\title{
An Equivalent Circuit for Collapse Operation Mode of CMUTs
}

\author{
Selim Olcum ${ }^{1}$, F. Yalcin Yamaner ${ }^{2}$, Ayhan Bozkurt ${ }^{2}$, Hayrettin Köymen ${ }^{1}$, and Abdullah Atalar ${ }^{1}$ \\ ${ }^{1}$ Bilkent University, Electrical and Electronics Engineering Department, Ankara, Turkey \\ ${ }^{2}$ Sabanci University, Electronics Engineering, Istanbul, Turkey
}

\begin{abstract}
Collapse mode of operation of the capacitive micromachined ultrasonic transducers (CMUTs) was shown to be a very effective way for achieving high output pressures. However, no accurate model exists for understanding the mechanics and limits of the collapse mode. In this work, we extend the analyses made for CMUTs working in uncollapsed mode to collapsed mode. We have developed an equivalent nonlinear electrical circuit that can accurately simulate the mechanical behavior of a CMUT under any large signal electrical excitation. The static and dynamic deflections of a membrane predicted by the model are compared with the finite element simulations. The equivalent circuit model can estimate the static deflection within $1 \%$ and the transient behavior of a CMUT membrane within $3 \%$ accuracy. The circuit model is also compared to experimental results of pulse excitation applied to fabricated collapse mode CMUTs. The model is suitable as a powerful design and optimization tool for the collapsed as well as the uncollapsed case of CMUTs.
\end{abstract}

\section{INTRODUCTION}

The collapse state of a suspended membrane is introduced for increasing the power output capability of capacitive micromachined ultrasonic transducers (CMUT) [1], [2]. However, only experiments and finite element method (FEM) simulations [3] have been utilized to understand the behavior of such a mode. Therefore, accurate models are still needed to be used in design and modelling. Conventionally, modelling efforts of CMUTs are based primarily on finite element (FEM) simulations and equivalent circuit models. In addition to the long simulation times, FEM simulations require a high level of complexity for accurate results, which makes them unsuitable for use in design and optimization. Electrical equivalent circuit models, on the other hand, are based on Mason's linear equivalent circuit model for the uncollapsed mode, which are inadequate to predict the collapse state. In recent years, the efforts for modelling the nonlinear behavior of CMUTs [4], [5] have increased. However, all those models are valid for the uncollapsed mode, as well.

In this paper, we introduce an electrical equivalent circuit model that can be used to simulate the behavior of a CMUT under any electrical excitation. The model can predict the membrane movement in the collapsed state as well as in the uncollapsed state of a CMUT. The simulation results of the circuit for static and dynamic deflections are compared to the electrostatic and transient FEM simulation results. Acoustical pressure generated at the surface of a CMUT membrane under a large pulse excitation is calculated by the circuit model and compared to transient FEM simulation and experimental results.

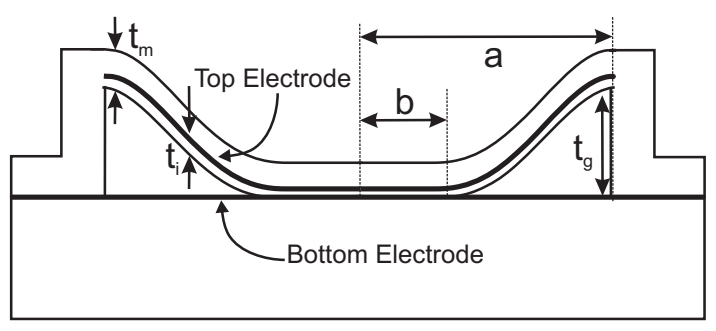

Fig. 1. Cross section of a collapsed clamped circular membrane with radius $a$, thickness $t_{m}$ and gap height of $t_{g}$. The top electrode is at a distance $t_{i}$ above from the gap. $b$ is the contact radius. The bottom electrode is assumed to be the top of the substrate.

\section{Equivalent Circuit Model}

As an electric potential is applied between the top electrode and the bottom electrode of a CMUT, the membrane deflects due to the electrostatic forces. Applied electrostatic forces result in a transmission of a pressure signal. It has been demonstrated in previous studies [1] that the collapsed mode increases the pressure amplitude drastically. More recently, it has been shown that a full electrode coverage should be utilized with the collapsed mode [2] for highest pressure amplitudes at the output. Therefore both of the electrodes of the CMUTs considered in this paper have full electrode coverage over the membrane region. A cross-sectional view of a collapsed membrane is illustrated in Fig. 1.

For the uncollapsed case, the displacement profile, $x(r)$, as a function of applied uniform pressure is given by [4] or [5]. For the collapsed case, on the other hand, the displacement profile under uniform pressure is given by [2]. Although the electrostatic force on a CMUT membrane is not uniformly distributed in the case of an applied potential, we assume that any deflection profile caused by the applied voltage can be approximated by the deflection caused by a uniform pressure as in [4]. This is a good assumption when the membrane has an electrode covering its full surface. We use the root mean square (rms) displacement, $x_{r m s}$, integrated over the surface of the membrane as the lumped displacement measure. The restoring force of a membrane as a function of its rms displacement under a uniformly distributed load is depicted as the solid line in Fig. 2. Additionally, the electrical force generated by a voltage can be determined by using the deflection profiles in [2] and the equations in [4] or [5] as a function of the rms displacement. The resulting electrical force curves with respect to the rms displacement are plotted for different voltages in 


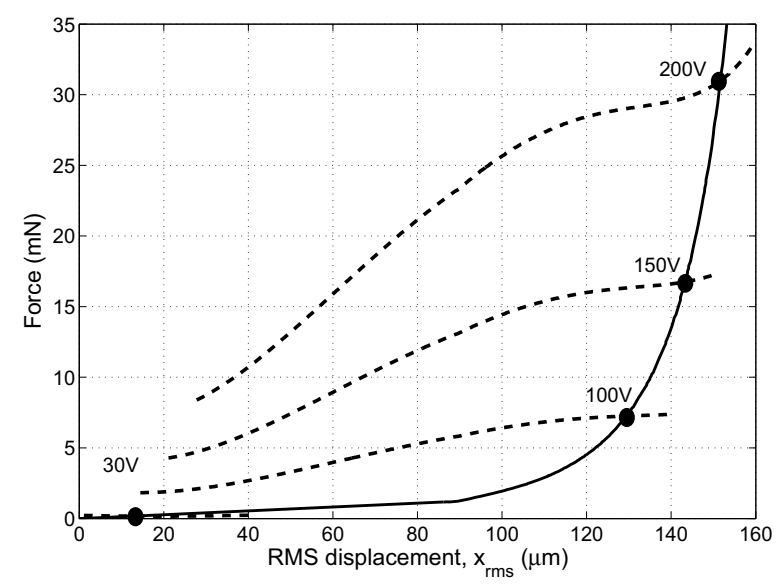

Fig. 2. Mechanical restoring force (solid) and electrostatic forces when the bias is $50,100,150$ and 200 Volts as a function of rms displacement, $x_{r m s}$. The membrane under consideration has a radius of $30 \mu \mathrm{m}$ and a thickness of $1.4 \mu \mathrm{m}$. The buried electrode covering the full surface is $0.4 \mu \mathrm{m}$ away from $0.2 \mu \mathrm{m}$ thick gap.

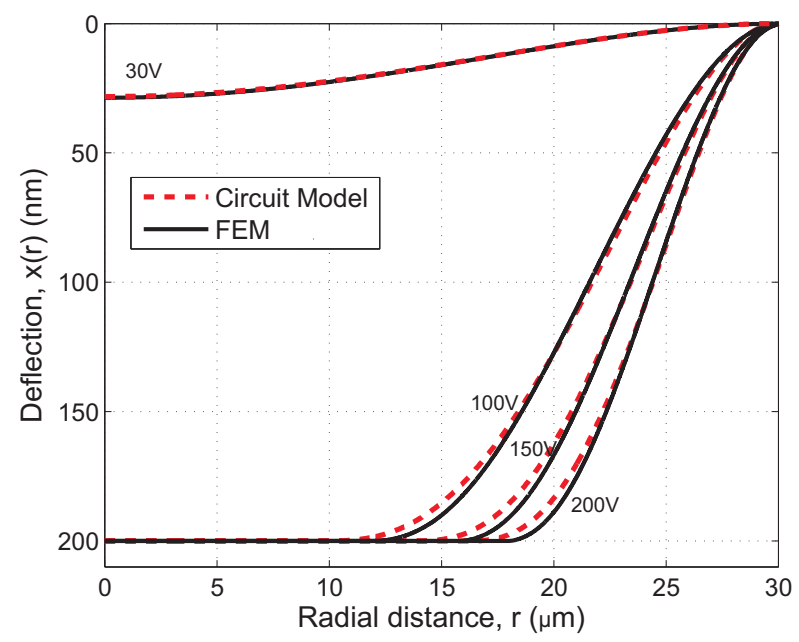

Fig. 3. Displacement profile of a membrane with a radius of $30 \mu \mathrm{m}$ and a thickness of $1.4 \mu \mathrm{m}$ for different values of bias. The profiles calculated by the numerical method (dashed) are compared to FEM simulation results (solid). The buried top electrode of the CMUT is $0.4 \mu \mathrm{m}$ away from $0.2 \mu \mathrm{m}$ thick gap.

Fig. 2 (dashed curves).

Although a closed form expression cannot be determined for the deflection profile of a membrane under the excitation of an electric potential, a numerical solution is possible by determining the intersection of the nonlinear electrical and restoring force curves. The intersection points of different electrical force curves with the restoring force curve in Fig. 2 give the equilibrium displacement points, when the atmospheric pressure is neglected. In Fig. 3, the profiles calculated by the proposed numerical method is compared to the profiles obtained by electrostatic FEM simulations for different bias values. The effect of the atmospheric pressure can be easily included by adding a constant force term in addition to the electrostatic force.

Previously developed models in the literature fail at the point of collapse. The method in this paper, however, can

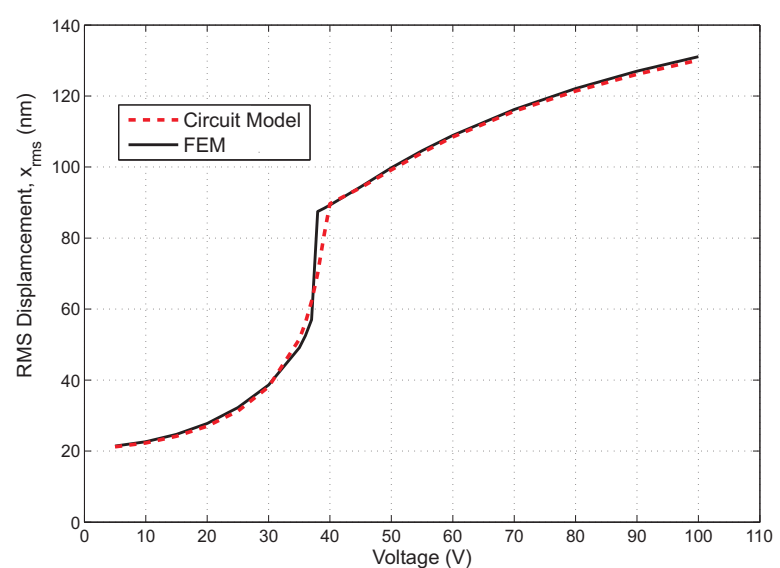

Fig. 4. RMS displacement of a membrane with a radius of $30 \mu \mathrm{m}$ and a thickness of $1.4 \mu \mathrm{m}$ as a function of the applied voltage. The displacements calculated by the numerical method (circles) are compared to FEM simulation results (solid). The buried top electrode of the simulated CMUT is $0.4 \mu \mathrm{m}$ away from $0.2 \mu \mathrm{m}$ thick gap. The effect of the atmospheric pressure is included.

predict and model the deflection before and after the collapse of the membrane. In Fig. 4, $x_{r m s}$ as a function of applied voltage is shown in comparison to FEM simulation results. The error between our prediction and the electrostatic FEM simulation results is less than $1 \%$, except for voltages in the close vicinity of the collapse voltage. The increased error near the collapse voltage can be attributed to the deviation of the profile from that generated by a uniformly distributed load.

The two forces, which are depicted in Fig. 2, and the force generated by the atmospheric pressure can be modelled in an electrical equivalent circuit as voltage sources connected in series as illustrated in Fig. 5. The force generated by the atmospheric pressure is represented by a DC source, $V_{a t m}$, with a value of the atmospheric pressure, $P_{0}$ times the membrane area:

$$
V_{a t m}=P_{0} \pi a^{2}
$$

In the electrical circuit, the rms membrane velocity, $v_{r m s}(t)$, is represented by the current, $i_{v}(t)$, and the rms membrane displacement, $x_{r m s}(t)$, is represented by the corresponding charge, $q_{x}(t)$. The electrostatic force on the membrane is a function of the applied voltage as well as the position of the membrane. Hence, the electrostatic force is modelled by a voltage and charge controlled nonlinear voltage source, $V_{e l}\left(V_{i n}, q_{x}\right)$. Electrostatic force curves as a function of rms displacement can be seen as the dashed lines in Fig. 2. It has been shown in [2] that electrostatic force, $F_{e}$ is proportional to the square of the applied voltage. Hence, we approximate the nonlinear electrostatic force curve using a sixth degree polynomial as

$$
V_{e l}\left(V_{i n}, q_{x}\right)=V_{i n}^{2}\left(\alpha_{0}+\alpha_{1} q_{x}+\alpha_{2} q_{x}^{2}+\ldots+\alpha_{6} q_{x}^{6}\right)
$$

where the coefficients $\alpha_{0}, \alpha_{1} \cdots \alpha_{6}$ are found to give a best fit to the dashed curve of Fig. 2.

The restoring force, on the other hand, is a function of membrane displacement and therefore it is modelled by a charge controlled nonlinear voltage source, $V_{\text {res }}\left(q_{x}\right)$. As seen 


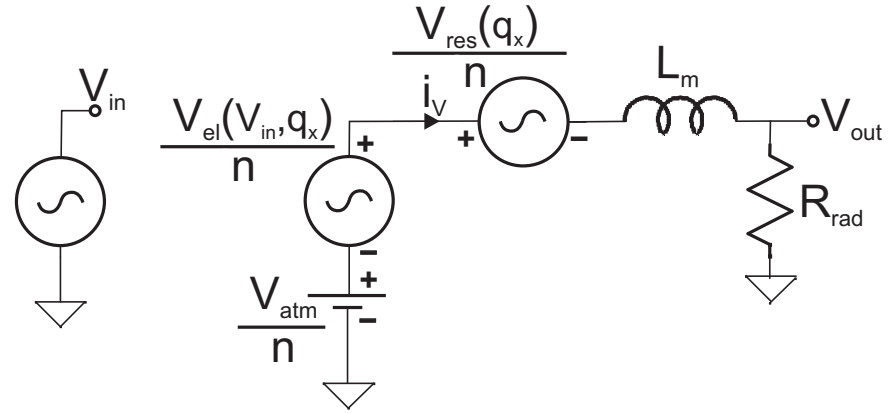

Fig. 5. Equivalent circuit model for simulating the behavior of clamped circular CMUTs.

in Fig. 2, the restoring force curve as a function of $x_{r m s}$ can be divided into two regions: uncollapsed and collapsed. A membrane under uniform pressure touches the substrate when $x_{r m s}=t_{g} / \sqrt{5}$. In the uncollapsed region, the total force on the membrane is linearly proportional to $x_{r m s}$. On the other hand, in the collapse region the restoring force as a function of $x_{r m s}$ is nonlinear and can be approximated by a third order polynomial. We write the charge controlled nonlinear voltage source, $V_{\text {res }}$, representing the restoring force of the membrane as follows

$$
V_{\text {res }}\left(q_{x}\right)= \begin{cases}\frac{64 \sqrt{5} \pi D}{a^{2}} q_{x}, & q_{x} \leq \frac{t_{g}}{\sqrt{5}} \\ \beta_{0}+\beta_{1} q_{x}+\beta_{2} q_{x}^{2}+\beta_{3} q_{x}^{3}, & q_{x} \geq \frac{t_{g}}{\sqrt{5}}\end{cases}
$$

where the coefficients $\beta_{0}, \beta_{1} \cdots \beta_{3}$ are found to give a best fit to the solid curve of Fig. 2. Since we use rms displacement as the through variable in the circuit, the voltages (representing forces) should be properly scaled [2] using the following factor:

$$
n= \begin{cases}\frac{3}{\sqrt{5}} & \text { for } x_{r m s}<\frac{t_{g}}{\sqrt{5}} \\ \frac{3}{\sqrt{5}}-\gamma_{0}\left(x_{r m s}-\frac{t_{g}}{\sqrt{5}}\right) & \text { for } x_{r m s}>\frac{t_{g}}{\sqrt{5}}\end{cases}
$$

where $\gamma_{0}$ is calculated using the deflection profiles in the collapsed state. The dynamic problem can be solved by modelling the mass of the membrane and the loading of the immersion medium as circuit components similar to Mason's circuit model. The mass, $m$, of the membrane is represented by a series inductor [5], $L_{m}$, with a value equal to the mass of the membrane:

$$
L_{m}=\pi a^{2} \rho t_{m}
$$

where $\rho$ is the density of the membrane material.

The radiation impedance of a circular, clamped membrane of radius $a$ for the uncollapsed mode was calculated earlier [6]. It was shown that for large $k a$ values, the radiation impedance approaches to

$$
Z_{\text {rad }}=\pi a^{2} \rho_{0} c \quad \text { for large } k a
$$

It turns out this expression is also valid for the collapsed case even with a finite contact radius $b$ [7].

\section{RESULTS}

We have tested the results of our equivalent circuit model by comparing them to FEM simulation and experimental

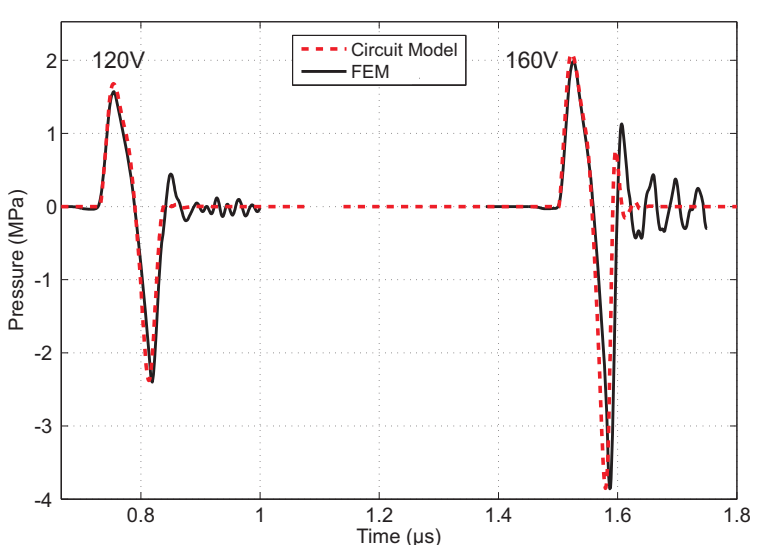

Fig. 6. Comparison of the calculated average pressure output using FEM (solid) and equivalent circuit (dashed) simulation results. The simulated CMUT has $30 \mu \mathrm{m}$ radius and $1.4 \mu \mathrm{m}$ thickness. The gap height is $200 \mathrm{~nm}$ and the insulation layer thickness is $400 \mathrm{~nm}$.

results. The developed equivalent circuit is implemented using LTSpice circuit simulator, which is a public domain software. The coefficients of the polynomials representing the nonlinear functions of the dependent voltage sources are imported to SPICE as parameters calculated externally by a MATLAB code.

We applied negative 120 and 160 Volts pulses to a CMUT with $\sim 35 \mathrm{~V}$ of collapse voltage. These pulses are applied on top of a positive DC bias with the same amplitude. The rise and fall times of pulse are $20 \mathrm{~ns}$ and the pulse width is $40 \mathrm{~ns}$. The resulting pressure amplitudes calculated by the equivalent circuit simulations are plotted in Fig. 6 (dashed) along with the FEM simulation results (solid). The peak-to-peak amplitude of the transmitted pressures are predicted within $3 \%$ and the pulse width of the prominent cycle is estimated within 5\% accuracy in the worst case. The low frequency oscillation seen in the FEM results is a spurious oscillation as a result of rigidly defined stand regions and lossless nature of the simulation.

The radiation resistance in (6) is used in the equivalent circuit model, assuming the membranes are fully loaded by the medium. This assumption is true for the membranes at the center of a large array. This assumption is also true for a membrane in a rigid baffle as in the case of FEM simulations. However, for a fabricated CMUT array, not all the membranes are at the center part of the array. Therefore, the equivalent radiation impedance is somewhat lower. The radiation impedance for such an array of CMUT membranes is calculated in [6] for the uncollapsed mode. It is shown that the normalized radiation impedance approaches to $\frac{1}{2} \rho_{0} c$ when the array is sufficiently large. Therefore, for comparing the results of the equivalent circuit simulations to experimental results obtained from fabricated devices, we use half the radiation resistance of the fully loaded case as shown in [6].

The CMUT array has 11 by 11 cells with $5 \mu \mathrm{m}$ spacing with a grid distribution resulting with a $67 \%$ fill factor. The aperture size is $0.71 \mathrm{~mm}$ by $0.71 \mathrm{~mm}$. The fabrication process of the array is discussed in [2]. A comparison of the simulation results to experiments are depicted in Fig. 7 when the CMUTs 


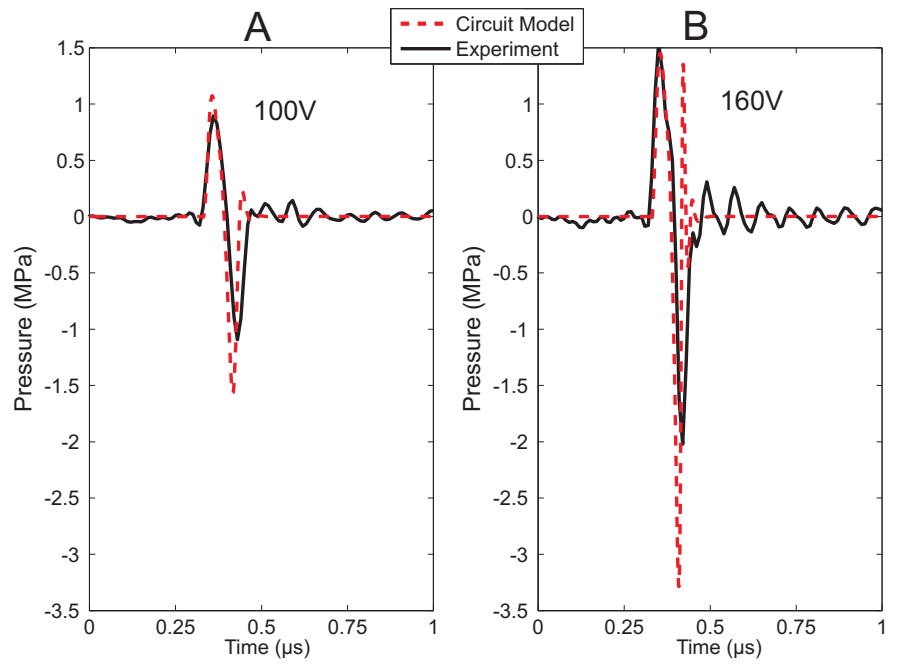

Fig. 7. Comparison of the equivalent circuit simulations (dashed) to experimental results (solid). The CMUTs used in the experiments have $30 \mu \mathrm{m}$ radius and $1.4 \mu \mathrm{m}$ thickness. The gap height is $200 \mathrm{~nm}$ and the insulation layer thickness is $400 \mathrm{~nm}$.

are excited with electrical signals of $120 \mathrm{~V}$ and $160 \mathrm{~V}$ negative amplitudes on equal amplitude positive DC biases. Although, the positive cycles of the output pressure are well predicted by the equivalent circuit simulations, the negative cycles are faster in the circuit model, due to the course approximation for the radiation resistance. In order to achieve a higher accuracy for predicting the response of a complete array, a more accurate radiation impedance model is needed.

Using the developed model, we demonstrate the deep collapse mode of CMUTs. We choose a particular membrane radius and thickness and measure the generated pressure under a fixed voltage step as a function of gap height. Fig. 8 is a plot of the simulation results. The curves are for a membrane with $30 \mu \mathrm{m}$ radius and $1.4 \mu \mathrm{m}$ thickness. The applied electrical steps are $100 \mathrm{~V}$ in amplitude and have $10 \mathrm{~ns}$ rise and fall times. For gap heights larger than $420 \mathrm{~nm}$, the membrane does not go into the collapsed state and a pressure of $0.25 \mathrm{MPa}$ is obtained. On the other hand, for a gap height of about $60 \mathrm{~nm}$, a collapsing membrane generates a pressure output of $4 \mathrm{MPa}$, demonstrating the attractiveness of the deep collapse operation. Note that with such a small gap height CMUTs have very low collapse voltages in the order of 10 Volts.

We note that SPICE electrical equivalent circuit simulations take no longer than a few seconds. The results obtained are very good agreement with FEM simulations lasting approximately one hour for a single simulation. While it is very difficult to make optimizations with such tedious FEM simulations, optimization of CMUT dimensions are easily done with the electrical equivalent circuit model.

\section{CONCLUSIONS}

In this work, we have developed a nonlinear equivalent circuit model for CMUTs and performed transient analyses of the equivalent circuit using a SPICE simulator. Exact analytical equations are used to determine the relation between the static deflection of a CMUT membrane and the forces

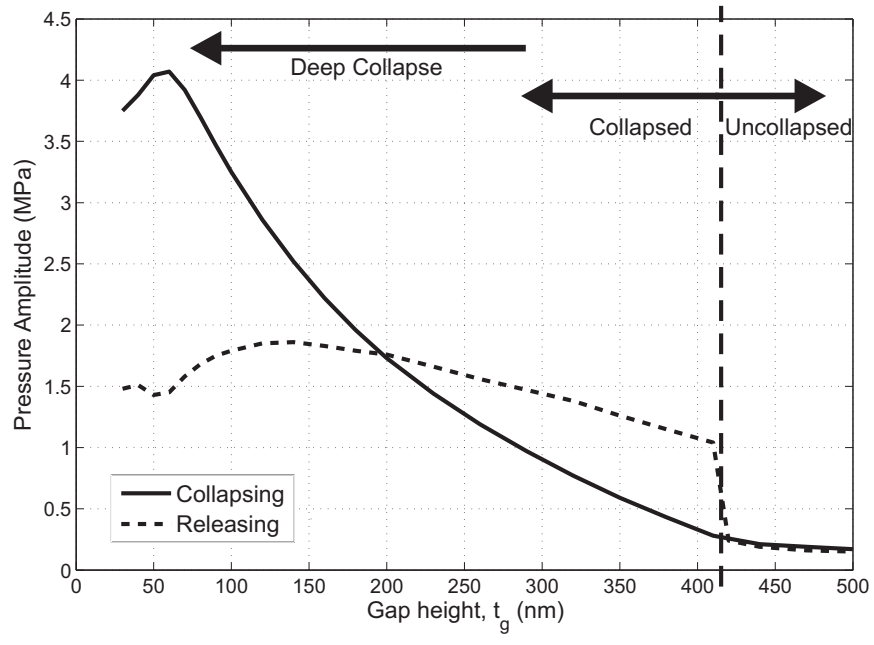

Fig. 8. Peak pressure output of a CMUT array with a step excitation of $100 \mathrm{~V}$ amplitude as a function of the gap height as found from the electrical equivalent circuit. Considered membranes has $30 \mu \mathrm{m}$ radius and $1.4 \mu \mathrm{m}$ thickness.

acting on the membrane. The two opposing forces are the electrical force acting toward the substrate and the restoring force of the membrane. The resulting equivalent circuit can predict the pressure output of a CMUT membrane with a very good accuracy.

The equivalent circuit model can be enhanced further for modelling fabricated CMUT arrays. Incorporating a better radiation impedance model to the circuit would improve the accuracy.

\section{ACKNOWLEDGEMENTS}

This work was supported by TUBITAK under project grant 107T921 and could not be possible without the concluded grants 105E023 and 104E067. S.O. gratefully acknowledge the financial support of TUBITAK and ASELSAN A.Ş. for their National Scholarship and Ph.D. Fellowship Programs, respectively. A.A. acknowledges the support of TUBA.

\section{REFERENCES}

[1] Ö. Oralkan, B. Bayram, G. G. Yaralioglu, A. S. Ergun, M. Kupnik, D. T. Yeh, I. O. Wygant, and B. T. Khuri-Yakub, "Experimental characterization of collapse-mode CMUT operation," IEEE Trans. Ultrason., Ferroelect., Freq. Contr., vol. 53, pp. 1513-1523, 2006.

[2] S. Olcum, F. Y. Yamaner, A. Bozkurt, H. Köymen, and A. Atalar, "Deep collapse operation of capacitive micromachined ultrasonic transducers," Submitted., 2010.

[3] B. Bayram, G. G. Yaralioglu, M. Kupnik, A. S. Ergun, Ö. Oralkan, A. Nikoozadeh, and B. T. Khuri-Yakub, "Dynamic analysis of capacitive micromachined ultrasonic transducers," IEEE Trans. Ultrason., Ferroelect., Freq. Contr., vol. 52, pp. 2270-2275, 2005.

[4] I. O. Wygant, M. Kupnik, and B. T. Khuri-Yakub, "Analytically calculating membrane displacement and the equivalent circuit model of a circular cmut cell." in Proc. IEEE Ultrason. Symp., 2008, pp. 2111-2114.

[5] H. K. Oguz, S. Olcum, M. N. Senlik, V. Tas, A. Atalar, and H. Köymen, "Nonlinear modeling of an immersed transmitting capacitive micromachined ultrasonic transducer for harmonic balance analysis," IEEE Trans. Ultrason., Ferroelect., Freq. Contr., vol. 57, pp. 438-447, 2010.

[6] M. N. Senlik, S. Olcum, H. Köymen, and A. Atalar, "Radiation impedance of an array of circular capacitive micromachined ultrasonic transducers," IEEE Trans. Ultrason., Ferroelect., Freq. Contr., vol. 57, pp. 969-976, 2010.

[7] P. R. Stepanishen, "Impulse response and radiation impedance of an annular piston,” J. Acoust. Soc. Am., vol. 56, pp. 305-312, 1974. 